\title{
CDISC SDTM Holter ECG Results Terminology
}

National Cancer Institute

\section{Source}

National Cancer Institute. CDISC SDTM Holter ECG Results Terminology. NCI Thesaurus. Code C120522.

Terminology associated with the Holter ECG results codelist of the Clinical Data Interchange Standards Consortium (CDISC) Study Data Tabulation Model (SDT M). 